\title{
Modeling Voice over LTE
}

\author{
Ahmed Amou El Arby and Ousmane Thiare \\ University Gaston Berger, Saint Louis, Senegal \\ E-mail: elarby.amou@gmail.com; othiare@ugb.edu.sn \\ Received 15 September 2016; Accepted 11 October 2016; \\ Publication 22 November 2016
}

\begin{abstract}
The 4G technology, known as Long Term Evolution (LTE), has dominated the telecommunication market the last few years due to its high data rate (up to $100 \mathrm{Mbps}$ ) that will perfectly satisfy the users multimedia applications needs in term of bandwidth and that rely on IP domain network. However, LTE lack in offering services for applications that rely on Circuit Switched (CS) domain particularly the voice CS service. This paper studies the latest up to date solutions for the voice over LTE problem such as Single Radio Voice Call Continuity, Circuit Switched Fall Back and Voice over LTE via Generic Access. The paper analyzes the pros and cons for these solutions and concludes with a suggestion of the best suitable solution for the operators to provide a seamless voice over LTE based on their infrastructure. Also, this paper models the long term solution and proposes an analytical model for simulating the performance of Voice over LTE (VoLTE) that can be used to simulate the behavior of such solution.
\end{abstract}

Keywords: LTE, SRVCC, VoLGA, CSFB.

\section{Introduction}

Traditionally, in the second and third generation $(2 \& 3 \mathrm{G})$ mobile networks the data has been provided through the Packet Switched (PS) domain and the voice through the Circuit Switched (CS) domain. As an evolution of the 3GPP

Journal of Software Networking, 101-112.

doi: 10.13052/jsn2445-9739.2016.006

(c) 2016 River Publishers. All rights reserved. 
networks e.g $2 / 3 \mathrm{G}$ the LTE- $4 \mathrm{G}$ come to live providing a high data rates up to $100 \mathrm{Mbps}$ (downlink) and 50 Mbps (uplink). The emphasis in LTE has been given to the PS domain since the growth of the data applications on mobile and their greedy need of the bandwidth and speed; as a result the LTE has only PS domain support.

The absence of the CS domain in LTE raise the issue of providing the voice call over LTE which consist of $80 \%$ of operators revenue [7] and ensure the continuity with the legacy networks $(2 / 3 \mathrm{G})$.

This paper analyzes the various up to date solutions in the telecommunication market such as the Circuit Switched Fallback [1, 2], Voice over LTE via Generic Access (VoLGA) [3], the Single Radio Voice Call Continuity (SRVCC) [4, 5] and Third Party Voice Over IP [6].

The paper presents and compares these solutions based on the impact on the network infrastructure, the solution complexity and the scalability of the solution to help the operators in eventual decision situation.

The rest of the paper is structured as follow: In Section 2, we review widely the existing solutions such as Circuit Switched Fallback), the voice over LTE via the Generic Access, the soft solution and the 3GPP target and long term solution of providing single voice call continuity. In Section 3, we compare all the solutions while giving their pro and cons. Then and in the last section, we focus on the long term solution SRVCC and propose an analytical model that represents the handover delay interruption for simulating the performance of SRVCC.

\section{Voice over LTE Short Term Solutions}

\subsection{Circuit Switched FallBack (CSFB)}

The Circuit Switch Fallback is a short term solution that relies on the existing 2G/3G network. When a User Equipment (UE), camped on LTE, wants to make a CS call, it activates an Extended Service Request (ESR) to the Mobile Management Entity (MME). After receiving the ESR, the MME order the eNodeB to redirect the call to the legacy network $2 \mathrm{G} / 3 \mathrm{G}$. The UE receives the LTE Release Connection and camp on the legacy 2G/3G network and establishes the standard 2G/3G RRC Connection. The downside of this solution is the introduction of the delay due to the procedure execution. This delay varies based on the mobile originating the call, in this case the delay is around $4.7 \mathrm{~s}$ [1], or terminating the call, in this case the delay is around $2.84 \mathrm{~s} \mathrm{[1].}$ 


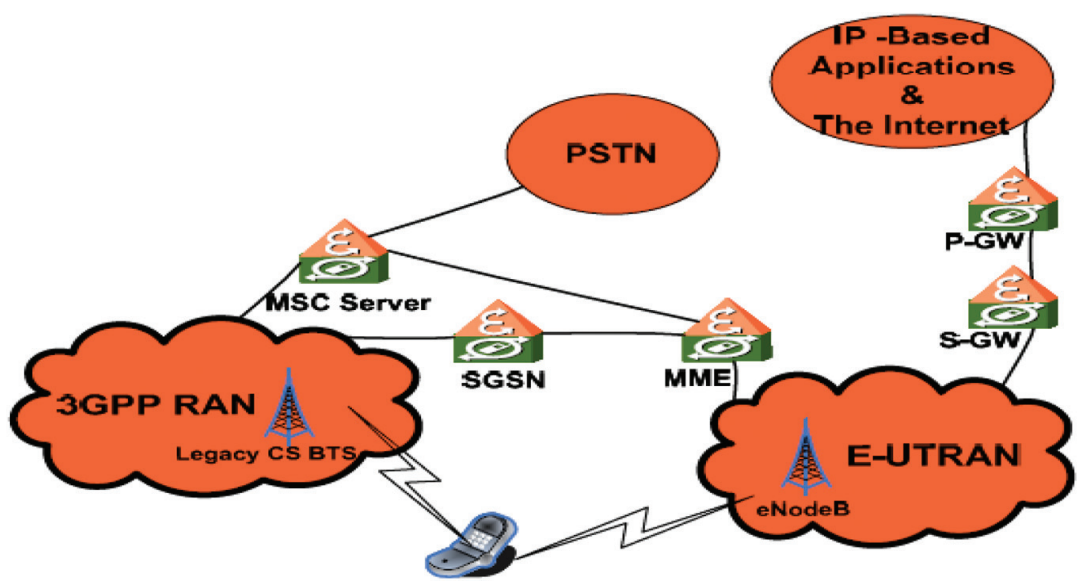

Figure 1 CSFB network architecture.

Furthermore, the CSFB requires an introduction to new interfaces between LTE and legacy $2 \mathrm{G} / 3 \mathrm{G}$ particularly the MME-MSC/SGSN and S-GW-SGSN [1] in order to perform the combined registration and paging process.

The benefit of using the CSFB is that the operators can use their existing $2 / 3 \mathrm{G}$ infrastructure to provide voice call.

\subsection{The Voice over LTE via Generic Acess (VoLGA)}

The Voice over LTE via Generic Access relies on the well-known Generic Access Network (GAN), which consists of adding a Wi-Fi radio interface to the UE becoming dual mode devices that can connect to Wi-Fi over a 2/3G network, when it is available, and connects to the network operator through a GAN gateway. Similarly, the VoLGA use the LTE as a replacement of the Wi-Fi with an introduction of one key critical entity named VoLGA Access Network Controller (VANC) which is a mix between an IP based node and a base station controller node from the LTE and GSM/UMTS point of view respectively. The VANC uses the same interfaces as $2 \mathrm{G}, 3 \mathrm{G}$ and $4 \mathrm{G}$ to connect with the mentioned technologies as per the Figure 2.

At the startup of the mobile, the UE register to the LTE and then establishes a secure IPSec tunnel connection to the VANC over LTE [3]. In addition, the UE register to the legacy network through the VANC secure connection that has just been established. The translation between the PS LTE and CS legacy is performed into the VANC entity and is completely transparent to the user and the network. 


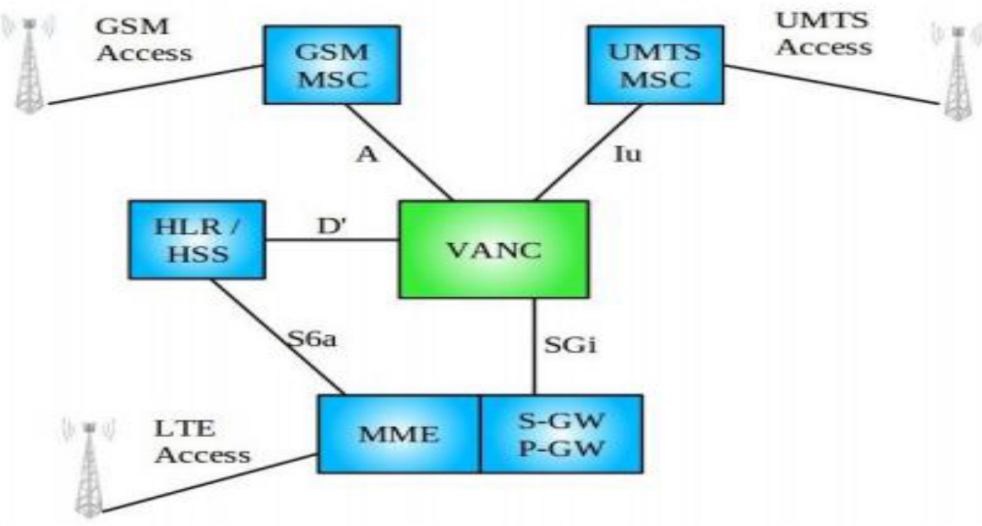

Figure 2 VoLGA network architecture.

Unlike the CSFB, the VoLGA doesn't impact the network entities MME, SGSN and MSC and doesn't increase the call setup procedure. Furthermore, VoLGA provides simultaneously the voice and data services to the UE. However, VoLGA has not been adopted by $3 \mathrm{GPP}$ and still requires all visited networks to support VoLGA in order to provide the roaming capability.

\subsection{Third Party Software for VoLTE}

Another solution that permits voice over LTE is enabled by using a third party software such as Viber or Skype, for providing calls over the LTE as presented in the Figure 3. No changes are required in the network [6], which makes the solution the least expensive among others VoLTE solutions. The downside of

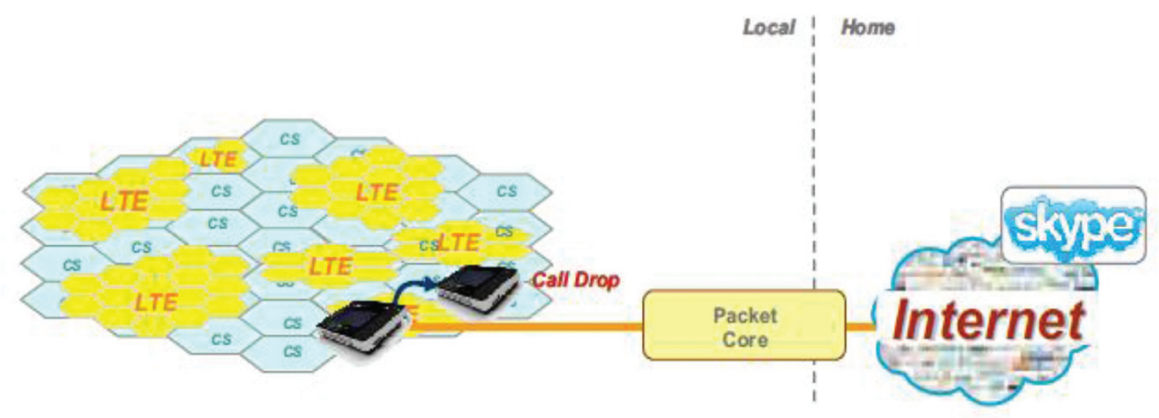

Figure 3 Voice over LTE via skype. 


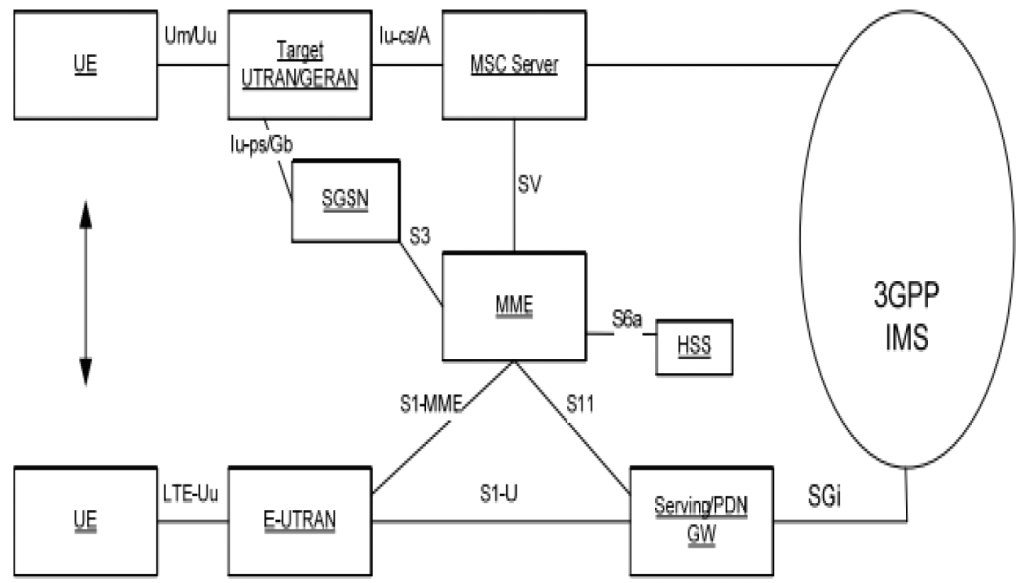

Figure 4 SRVCC architecture for E-UTRAN to UTRAN/GERAN.

this solution is that there is no quality of service guaranteed and this solution doesn't support the CS calls.

\subsection{Single Radio Voice Call Continuity}

The Single Radio Voice Call Continuity (SRVCC) is the 3GPP target solution, based on IMS, for ensuring the voice continuity between LTE and 2/3G networks. The SRVCC requires that the UE initiates the call using IMS and the Application Server that needs to be implemented in the IMS [4].

The SRVCC uses a combination of the IMS session continuity procedure with a handover procedure. In detail, when a UE reaches the border of the LTE coverage, the E-UTRAN will send a SRVCC handover request to the MME, the latest will trigger the SRVCC procedure with the enhanced MSC (eMSC). The eMSC will perform the session transfer to the IMS and coordinates it with the CS handover to the target cell. Finally the eMSC will send the Forward Relocation Response to MME, which includes the handover command. The Figures 4 and 5 presents the architecture and call flow of the SRVCC $[4,5]$.

\section{Solutions Summary and Comparaison}

Deploying a solution relies on the operators' current infrastructure and its future plans. Some operators choose the CSFB because of their existing legacy network infrastructure in place $(2 / 3 \mathrm{G})$, other adopt the VoLGA because the 


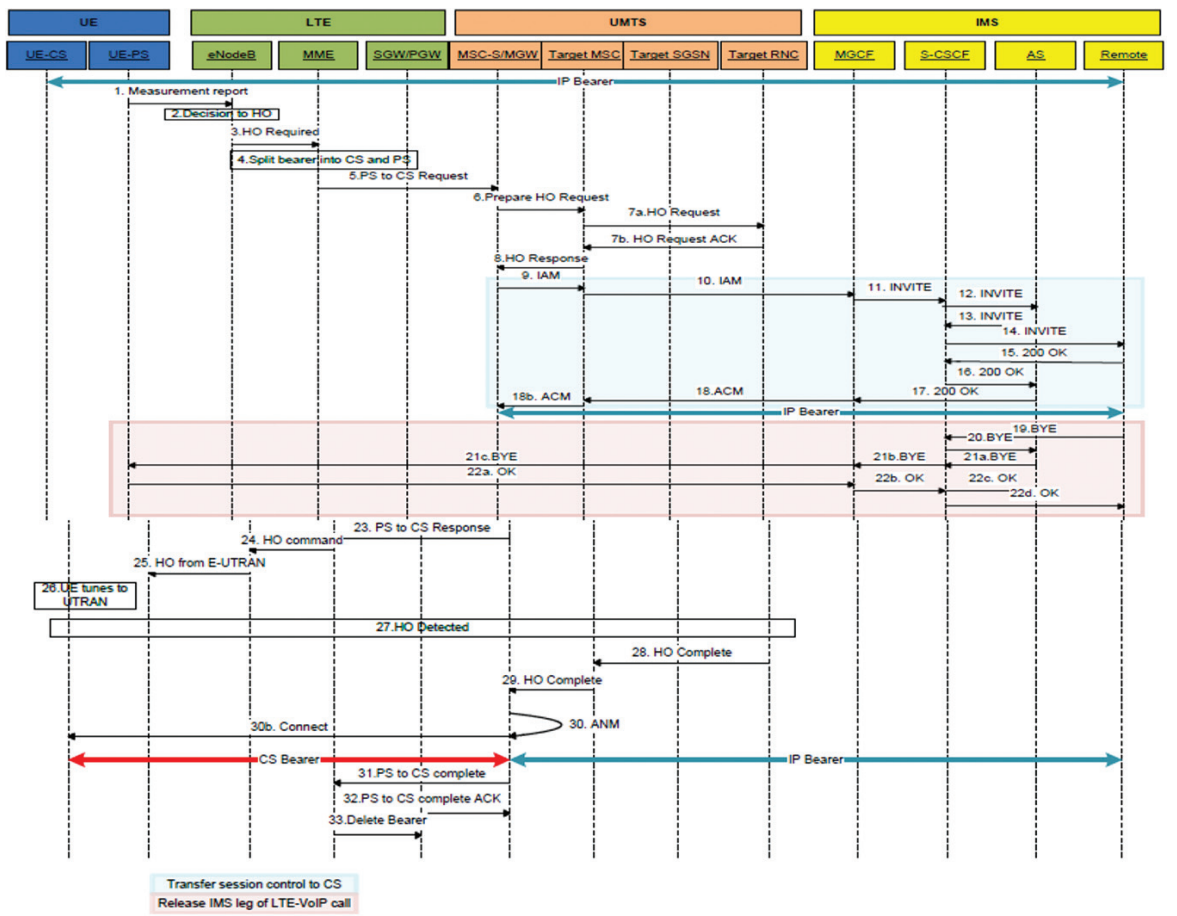

Figure 5 SRVCC call flow from E-UTRAN to UTRAN/GERAN.

availability of such a technology in their network. On the other hand and for a simple and costless, the third party solution will be chosen. For a durable, high and rich multimedia service, the solution based on IMS will be a suitable choice for the operator.

The Table 1 shows a comparison summary of the solutions:

Table 1 Voice over LTE scenarios

\begin{tabular}{|c|c|c|c|c|}
\hline & SRVCC & VoLGA & CSFB & Third Party \\
\hline $\begin{array}{l}\text { Availability of } 2 / 3 \mathrm{G} \text { and } \\
\text { neither GAN nor IMS } \\
\text { are available }\end{array}$ & No & $\begin{array}{l}\text { Yes, however } \\
\text { updates are } \\
\text { required at UEs } \\
\text { and an VANC } \\
\text { need to be } \\
\text { introduced }\end{array}$ & $\begin{array}{l}\text { Yes, however the } \\
\text { data portion will } \\
\text { be slightly } \\
\text { affected }\end{array}$ & $\begin{array}{l}\text { Yes, however no } \\
\text { QoS is guarantee }\end{array}$ \\
\hline $\begin{array}{l}2 / 3 \mathrm{G} \text { available and plans } \\
\text { for IMS }\end{array}$ & $\begin{array}{l}\text { Yes as long } \\
\text { term } \\
\text { solution }\end{array}$ & $\begin{array}{l}\text { Yes as an } \\
\text { alternative } \\
\text { solution }\end{array}$ & $\begin{array}{l}\text { Yes as a short } \\
\text { term solution }\end{array}$ & $\begin{array}{l}\text { Yes as an } \\
\text { alternative } \\
\text { solution with no } \\
\text { QoS }\end{array}$ \\
\hline
\end{tabular}


Table 1 Continued

\begin{tabular}{llll}
\hline $\begin{array}{l}\text { No 2/3G available and } \\
\text { plans for IMS }\end{array}$ & $\begin{array}{l}\text { Yes as long No } \\
\text { term } \\
\text { solution }\end{array}$ & No & No \\
$\begin{array}{l}\text { No 2/3G and no IMS } \\
\text { deployment plan }\end{array}$ & No No & No & Yes with no QoS \\
$\begin{array}{l}\text { Availability of GAN } \\
\text { Combination of VoLGA and } \\
\text { SRVCC for voice service } \\
\text { continuity }\end{array}$ & No & Yes \\
\hline
\end{tabular}

\section{Modeling SRVCC}

Many papers have studied the SRVCC handover and tried to come out with a standard model to simulate the performance of this long term solution for voice over LTE.

One of the mathematical models that have been developed is the model presented in [8], in which an analytical expression of the overall delay experienced by the call executing the handover is been presented. This delay was based on the sum of the delay caused by the radio link (RLC) and the delay caused by the remote and internet queuing which was assumed to be following the First In First Out queuing algorithm.

The results of [8] was more related to the radio link delay and has shown that the SRVCC handover interruption time will be reduce under a given Block Error Rate (BLER) with an increased data rate as presented in the Figure 6.

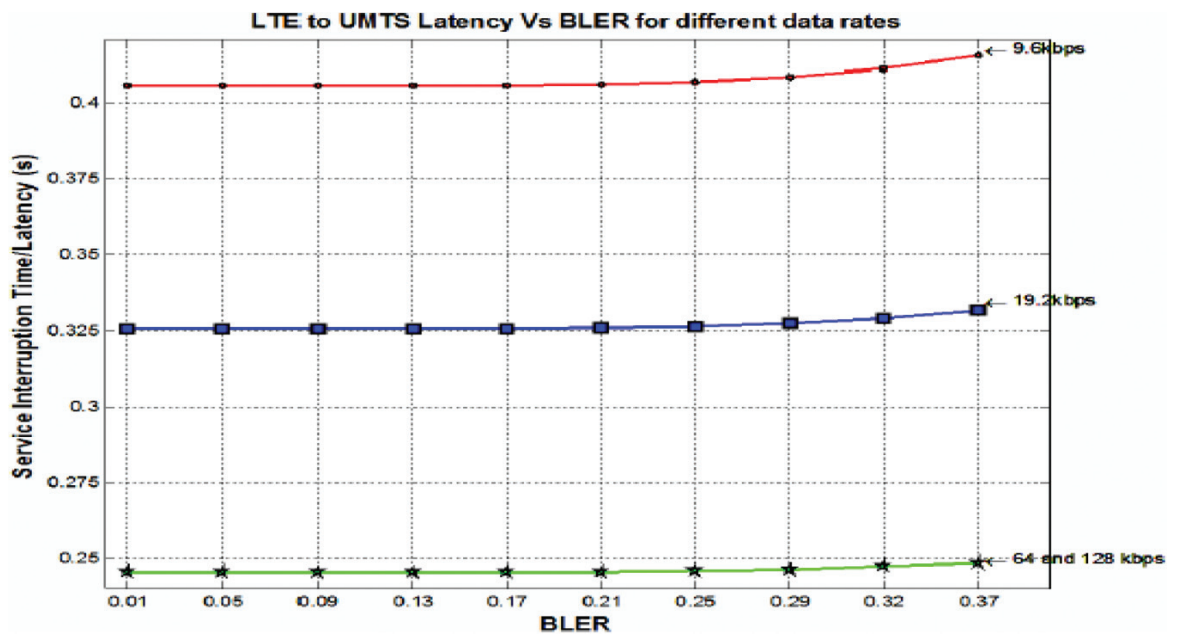

Figure 6 LTE to UMTS SRVCC service interruption time Vs BLER. 
In this paper, we will reevaluate the model while assuming that the Quality of Service (QoS) is applied in the whole network since it is crucial for LTE to implement the QoS in order to serve fairly the different traffics that flow through the network.

We estimate that applying a more advanced queuing algorithm will minimize the delay of the SRVCC packets in the network nodes and therefore reduce considerably the overall delay experienced by SRVCC handover which will enhance the performance of the handover.

In order to evaluate the performance of the SRVCC under priority queuing, it is necessary to analyze the interruption time experienced by the ongoing call. This delay, and based on the [9], can be split into three parts: Radio link delay, the network queuing delay and the remote network queuing delay. These delays can be represented by be mathematical models since they are following a unique mathematical behavior.

\subsection{The Radio Link Delay}

Based on several papers, the radio delay can be measured by analyzing the Radio Link Control (RLC). Assuming the same RLC model in LTE as UMTS, we can represent the delay in the radio part by the following Equation $1[8]$ :

$$
\begin{aligned}
T_{R L C}= & T_{\text {Iub }}+(k-1) T T I+\frac{k\left(P_{s}-(1-p)\right)}{P_{s}^{2}} \\
& *\left\{\sum_{j}^{n} \sum_{i}^{j}\left[P\left(C_{i j}\right)\left(2 j T_{\text {Iub }}+\left(\frac{j(j+1)}{2}+i\right) * T T I\right)\right]\right\}
\end{aligned}
$$

Equation 1 Radio Delay.

Where:

$\mathrm{k}$ : number of frames to be transmitted

$\mathrm{n}$ : number of RLC retransmissions

Ps: probability of receiving RLC frame successfully after $\mathrm{n}$ transmission

P: probability of RLC frame received erroneously

TIub: latency of the Iub interface

TTI: transmission time interval at eNodeB

$\mathrm{P}(\mathrm{Cij})$ : the first correctly received frame at destination 


\subsection{The Network Node and Remote Queuing Delay}

The delay caused by the nodes queues relies heavily on the algorithm applied in the system. Many algorithms has been developed and globally used such as FIFO, Priority Queuing (PQ) and Weight Round Robin PQ.

In this research and since LTE is an ALL-IP network that must apply queuing algorithm that guarantee the Quality of Service (QoS) in the network in delivering the traffic, we, first, assume that PQ algorithm has been applied in order to priorize packet coming from the handover process and therefore reducing their treatment delay in the queue.

In order to estimate the average waiting time (delay) in the queues for both the network queuing delay and for the remote queuing delay, we assume that the packets priority is $\mathrm{p}$ and arriving with a Poisson distribution with parameter $\lambda_{p}$ and their length has an exponential distribution with an average service time $\overline{x_{p}}$.

We can then calculate the average waiting time $W_{p}$ of priority p packets in the following way [10]:

$$
W_{p}=\frac{W_{0}}{\left(1-\sigma_{p}\right)\left(1-\sigma_{p+1}\right)}
$$

Equation 2 Average waiting time for PQ.

Where $W_{0}$ is the average delay caused by a packet, which is already serviced and calculated as in:

$$
W_{0}=\sum_{i=1}^{P} \frac{\lambda_{i} \overline{x^{2}}}{2}
$$

Where $\overline{x^{2}}$ is the second moment of service time.

And

$$
\sigma_{p}=\sum_{i=p}^{P} \rho_{i}=\sum_{i=p}^{P} \lambda_{i} \overline{x_{i}}
$$

After identifying the delay in each part of the involved part of the network, we will deduct the expression of the whole interruption delay experienced by a user that executes the SRVCC handover.

Based on the signal flow in the SRVCC in the Figure 5, we can notice that the service interruption start when the call is released in order to establish a new connection with the $3 \mathrm{G}$ network. The Figure 7 [8] represent a zoomed part of the signal flow during the interruption of the service. 


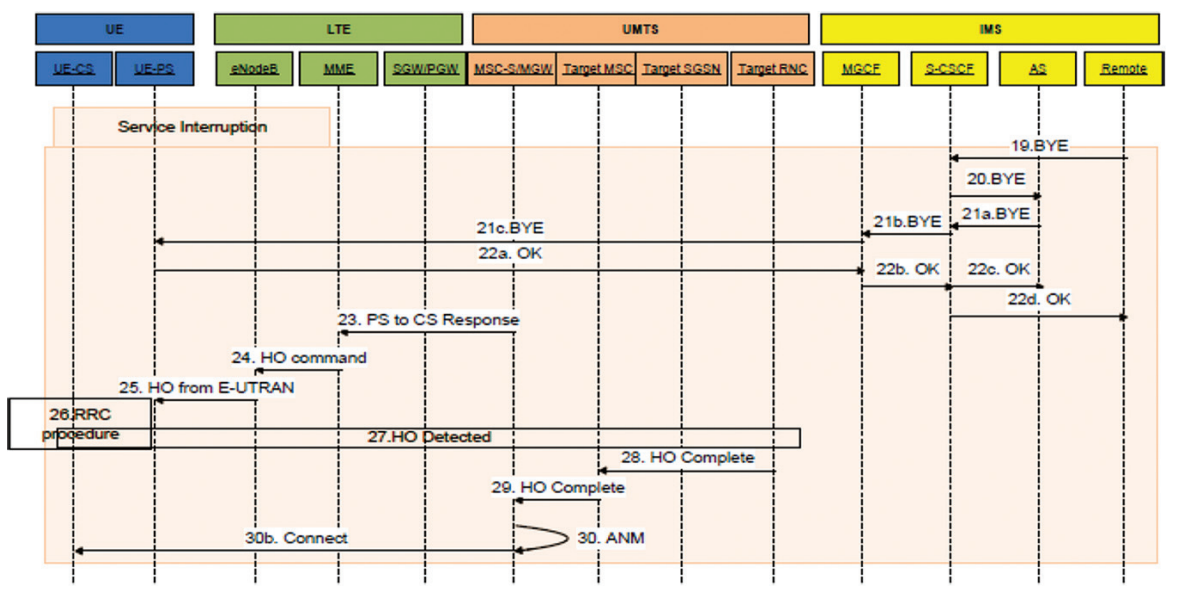

Figure 7 SRVCC service interruption.

Based on the figure and message flow in each node and radio link that is represented by each single arrow, we can write the equation that represent the overall interruption delay as follow:

$$
\begin{aligned}
\text { Dealy }_{S R C C}= & \text { Delay }_{\text {Remote }}+\text { Delay }_{\text {Internet }}+2 * \text { Delay }_{A S} \\
& +3 \text { Delay }_{C C F}+2 \text { Delay }_{M G C}+\text { Delay }_{U} \\
& +3 \text { Delay }_{M C}+\text { Delay }_{M E}+\text { Delay }_{\text {NodeB }} \\
& + \text { Delay }_{R C}+\text { Dleay }_{R C}
\end{aligned}
$$

Equation 3 Overall SRVCC interruption time.

Each value of the Equation 3 will be replaced by its expression that has already been presented in the Sections 1 and 2. The Equation 3 will be used to estimate the delay of the interruption of service of the ongoing call until the SRVCC handover has reestablish the circuit with the new cell. The delay will be evaluated under different parameters values in order to study the behavior of the SRVCC performance.

The simulation results and performances evaluation for different priority queuing will be presented in separate paper in the near future.

\section{Conclusion}

Providing voice calls in LTE has been a challenge and a necessity for the operators since LTE is designed for only PS and the significant incomes generated by the voice calls. Many temporary solutions has been proposed 
and used, however the operators still seeking for implementing the long term solution SRVCC that will enable the transmission of voice over LTE and supporting the CS over LTE. This solution has not been studied in depth in order to evaluate it performance. This paper has presented the different temporarily solution that are up to date and has focused on the long term solution while proposing a new analytical model that could be used to evaluate the performance of SRVCC. This model will be simulated via Matlab and that for different priority queuing in order to evaluate the performance of SRVCC under these conditions and will be a subject of another future publication.

\section{References}

[1] Vargas Bautista, J. E., Sawhney, S., Shukair, M., Singh, I., Govindaraju, V. K., and Sarkar, S. (2013). Performance of Fallback from LTE to UMTS Qualcomm Corporate Engineering. IEEE Commun Mag. 51, 136-143.

[2] CS fallback in Evolved Packet System. (2010). Technical Specification 3GPP TS 23.272.

[3] Voice over LTE with Generic Access. (2010). Technical Specification VoLGA Stage 2.

[4] Single Radio Voice Call Continuity. (2008). Technical Specification 3GPP TS 23.216.

[5] Single Radio Voice Call Continuity. (2010). Technical Specification 3GPP TR 23.856.

[6] Gavrilovic, S. (2010). "Standard Based Solutions for Voice SMS Services over LTE. IEEE/MIPRO 334-339.

[7] Cox, C. (2012). Introduction to LTE. Hoboken, NJ: Wiley.

[8] Namakoye, J., Van Olst, R. (2011). A Performance evaluation of a voice call handover scheme between LTE and UMTS. Rome: IEEE Conference Publications.

[9] Kim, K. (2009) "A seamless voice call handover scheme for next generation cellular network," in IEEE 15th Asia-Pacific Conference on Communications (Shanghai: IEEE).

[10] Balogh, T., and Medveck , M. (2010). Comparison of Priority Queuing Based Scheduling Algorithms. ElektroRevue. 


\section{Biographies}

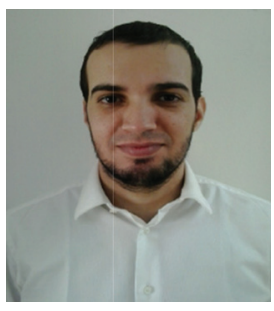

A. A. El Arby is a Ph.D. student at the University of Gaston Berger, Senegal since 2012. A Ph.D. that has been started at the Ecole de Technologie Superieure, Canada where he received his M.Sc. and Engineering diploma in telecommunication. He is Professor in Networking and Telecommunication, in Nouakchott University and ISCAE Mauritania. Author of published papers in several journals such as IEEE and Springer.

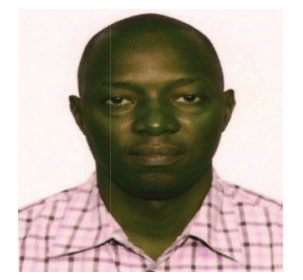

O. Thiare, received a Ph.D. in computer science (Distributed systems) at 2007 from the University of Cergy Pontoise, France. He is Full Professor in Computer science, in Gaston Berger University of Saint-Louis Senegal. He has been author and co-author of published papers in several journals and recognized international conferences and symposiums. 